\title{
Occupational exposure in lead and zinc mines induces oxidative stress in miners lymphocytes: Role of mitochondrial/lysosomal damage
}

https://doi.org/10.1515/mgmc-2020-0019

Received July 01, 2020; accepted July 24, 2020.

\begin{abstract}
The purpose of this research was to determine mitochondrial and lysosomal damage and oxidative stress status in blood lymphocytes of lead-zinc miners. This research was performed in 10 mine workers who have been in contact with lead and zinc in comparison to a control group containing 10 healthy volunteers. Lymphocytes were isolated from peripheral blood using the Ficoll standard method and then mitochondrial and lysosomal damage and oxidative stress were evaluated. The level of reactive oxygen species (ROS), collapse in the mitochondrial membrane potential (MMP) collapse, and glutathione disulfide (GSSG) content, and lysosomal damage in miners were higher than the control group. Also, viability and glutathione (GSH) content were decreased. The lymphocytes of workers of a lead-zinc mine are more susceptible to oxidative stress, mitochondrial and lysosomal damage. The proper use of safety equipment can reduce the risk of toxic agents and their subsequent hazards for mine workers.
\end{abstract}

Keywords: lead; zinc; mine workers; lymphocytes; heavy metal; oxidative stress

\footnotetext{
*Corresponding author: Jalal Pourahmad, Department of Pharmacology and Toxicology, School of Pharmacy, Shahid Beheshti University of Medical Sciences Tehran, Iran;

e-mail: j.pourahmadjaktaji@utoronto.ca

Enayatollah Seydi, Department of Occupational Health and Safety Engineering, School of Health, Alborz University of Medical Sciences, Karaj, Iran

Mahshid Soltani, Maral Ramazani and Mohammad Hadi Zarei, Department of Pharmacology and Toxicology, School of Pharmacy, Shahid Beheshti University of Medical Sciences Tehran, Iran
}

\section{Introduction}

In developing countries, heavy metals pollution is one of the serious problems and public awareness of their toxic effects is increasing (Qu et al., 2012). Occupational exposure is one of the most important sources of exposure to lead (Conterato et al., 2013; Ghanwat et al., 2016; Hsieh et al., 2017). The lead enter to the workers' body through the inhalation route, and then can accumulate in the vital organs of the body (Ghanwat et al., 2016; Hsieh et al., 2017; Kalahasthi and Barman, 2018). Inhalation, skin and ingestion are among the most important routes of exposure to zinc. According to the National Institute for Occupational Safety and Health (NIOSH), the number of workers exposed with zinc fumes is around 50,000 (Ricco et al., 2018). According to the Occupational Safety and Health Administration (OSHA), the blood lead level (BLL) in workers is less than $40 \mu \mathrm{g} / \mathrm{dL}$ (Basit et al., 2015).

Research has shown that heavy metals can increase the generation of reactive oxygen species (ROS) and oxidative stress through Fenton like reaction ( $\mathrm{Qu}$ et al., 2019). Oxidative stress is one of the most important mechanisms involved in lead toxicity (Kasperczyk et al., 2014b; Qu et al., 2019; Shraideh et al., 2018). In addition, lead can induce apoptosis signaling ( $Q u$ et al., 2019). It has been shown that lead has a high affinity to the sulfhydryl groups (SH) of proteins. Sulfhydryl groups play an important role in antioxidant enzymes against the generation of free radicals (Conterato et al., 2013; Kasperczyk et al., 2014a, 2016; Shraideh et al., 2018). An in vivo research has shown that overexposure to zinc affects the function of lymphocytes. Also, zinc excess has an effect on ROS generation, induction of oxidative stress, cytochrome c release, and induction of apoptosis (Plum et al., 2010). Exposure to lead leads to defects in lymphocyte function, and also changes in the ratio of lymphocytes subpopulation (Zarei et al., 2017). 
Mitochondria is the main source of ROS generation in the cell, and damage to the mitochondria causes excessive release of ROS and induction of oxidative stress (Zarei et al., 2018a). Sulfhydryl groups are located in all parts of the mitochondrial respiratory chain (Fedotcheva et al., 2012). Lead with a high affinity to sulfhydryl groups induces ROS generation and oxidative stress (Conterato et al., 2013; Kasperczyk et al., 2014a, 2016; Shraideh et al., 2018). The damage to the mitochondria can disrupt the mitochondrial membrane potential (MMP) and release the cytochrome $\mathrm{c}$ and eventually induce cell death. Disruption of the lysosomal membrane is due to hydrogen peroxide (H2O2) transmission of mitochondria to cytosol and activation of the fenton reaction (Zarei et al., 2018a). In this research, we aimed to study the oxidative stress marker and mitochondrial and lysosomal damage in relation to exposure to lead and zinc in peripheral blood lymphocytes taken from workers of a lead-zinc mine.

\section{Results and discussion}

\subsection{Cell viability}

The purpose of this study was to investigate the oxidative stress marker and mitochondrial and lysosomal damage in workers of a lead-zinc mine using blood lymphocytes. A few studies have been done on cytotoxic effects of heavy metals on human lymphocytes. Initially, the viability of lymphocytes of workers in the lead and zinc mine was evaluated. In this study, the survival of lymphocytes in
A

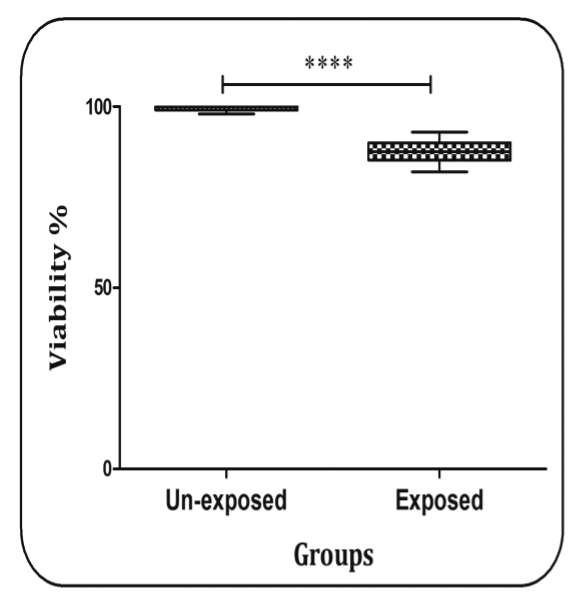

C

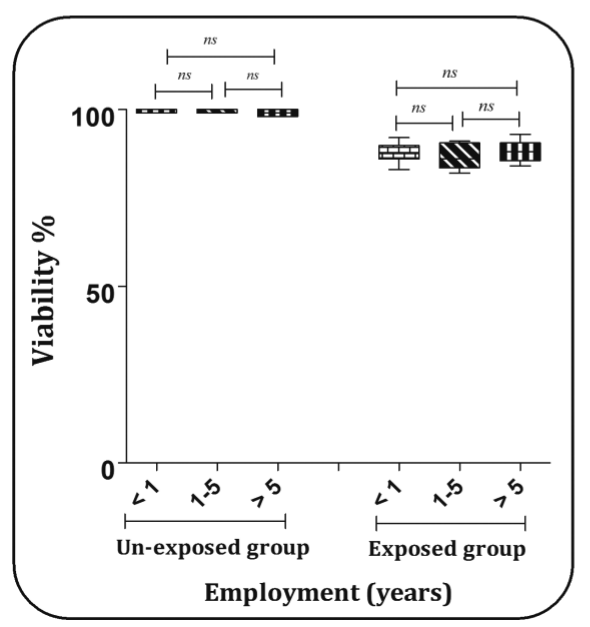

B

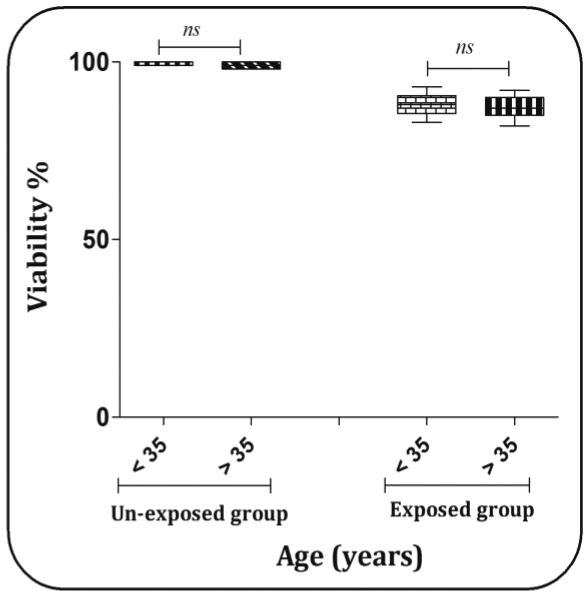

D

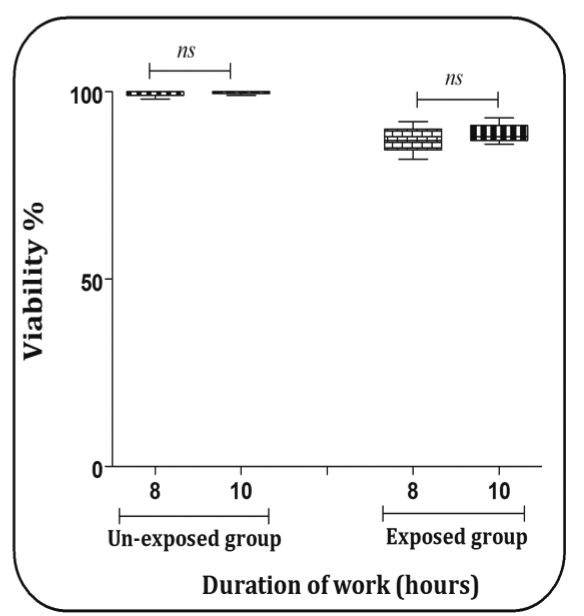

Figure 1: The cell viability assay. (a) Comparison between exposure and non-exposure groups. (b) Comparison between the exposed and unexposed groups based on age (year). (c) Comparison between the exposed and unexposed groups based on employment (year). (d) Comparison between the exposed and unexposed groups based on duration of work (hours). Data are presented as mean \pm SD $(\mathrm{n}=20){ }^{* \star * *} \mathrm{P}<0.0001$. 
exposed group is less than that of the un-exposed group (Figure 1a). These results are in agreement with previous studies shown the toxicity of lead and zinc in human lymphocytes using in vitro studies (Steffensen et al., 1994; Zarei et al., 2017, 2018a). In the exposed and un-exposed groups, there is a no significant difference in the cell viability based on the comparison of age, employment and duration of work (Figures $1 \mathrm{~b}-\mathrm{d}$ ).

\subsection{ROS generation}

In the blood lymphocytes isolated from the exposed group, the level of ROS generation is significantly higher than that of the un-exposed group. The results show that exposure to lead and zinc increases ROS generation in isolated lymphocytes (Figure 2a). In the exposed group, there is a significant difference in the level of ROS based on the comparison of age, employment and duration of work (Figures 2b-d).

Studies showed that excessive exposure to heavy metals such as lead and zinc increases the level of ROS and oxidative stress (Akram et al., 2018; Kasperczyk et al., 2017; Plum et al., 2010; Zarei et al., 2017, 2018a). Another study showed that the level of free radicals in workers exposed to lead was higher than that of the control group (Fracasso et al., 2002). A significant increase in ROS generation was observed in the blood lymphocytes isolated from the lead and zinc mine workers. Mitochondria (complexes 1 and III) are considered as one of the important sources in the generation of ROS. ROS play various roles in the different concentrations (Dan Dunn et al., 2015; Li et al., 2013; Zorov et al., 2014). It has been shown that heavy metals with an effect on mitochondria increase the generation of ROS

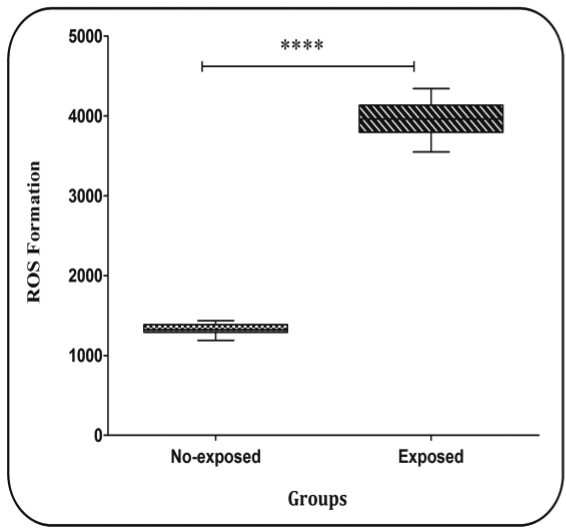

C

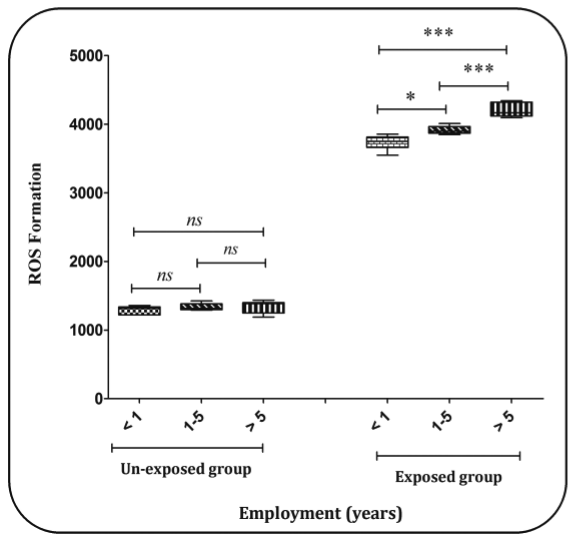

B

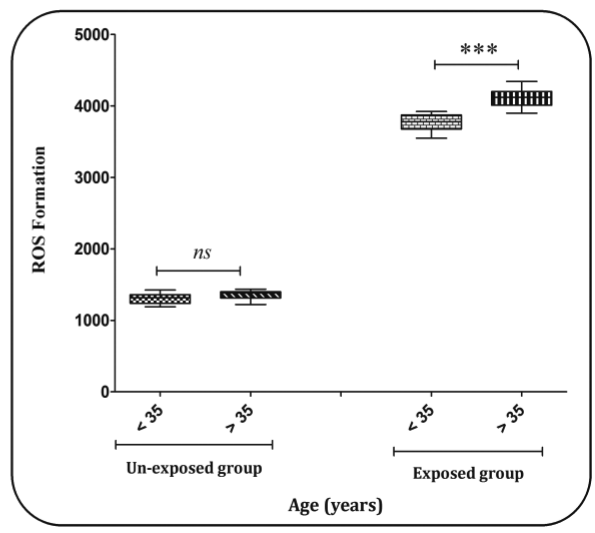

D

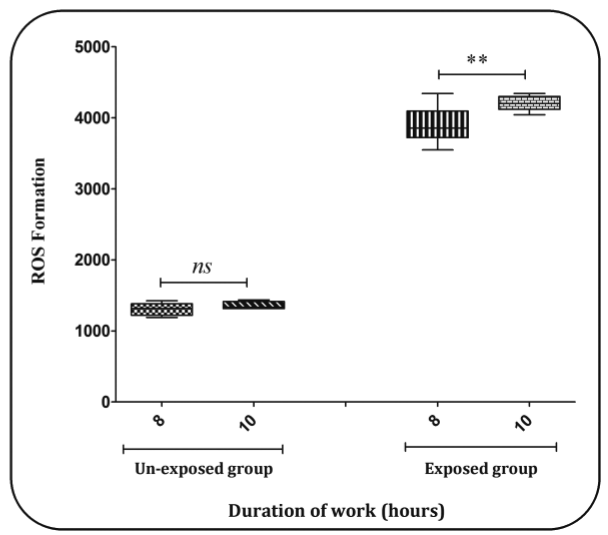

Figure 2: The mitochondrial ROS assay. (a) Comparison between exposure and non-exposure groups. (b) Comparison between the exposed and unexposed groups based on age (year). (c) Comparison between the exposed and unexposed groups based on employment (year). (d) Comparison between the exposed and unexposed groups based on duration of work (hours). Data are presented as mean \pm SD ( $n=20$ ). ${ }^{*} P<0.05$, ${ }^{\star *} P<0.01,{ }^{* *} P<0.001$ and ${ }^{* \star * *} P<0.0001$. The unit of measurement of ROS formation is based on fluorescent intensity. 
A

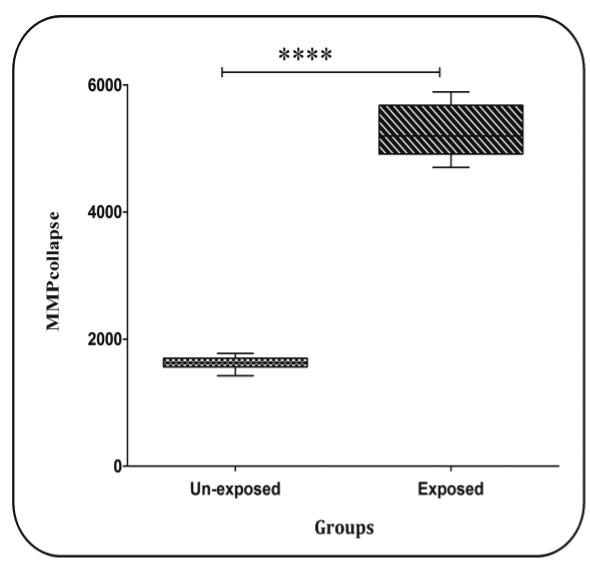

C

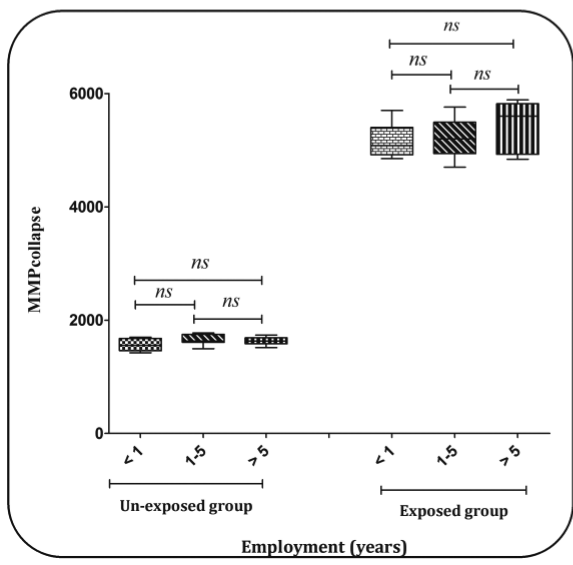

B

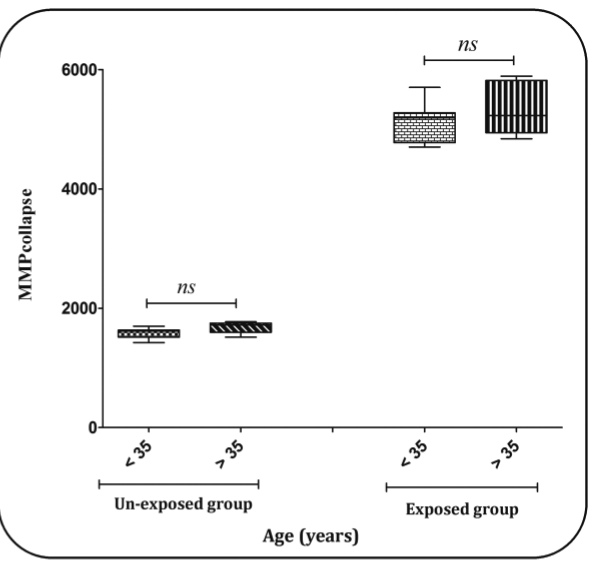

D

Figure 3: MMP assay. (a) Comparison between exposure and non-exposure groups. (b) Comparison between the exposed and unexposed groups based on age (year). (c) Comparison between the exposed and unexposed groups based on employment (year). (d) Comparison between the exposed and unexposed groups based on duration of work (hours). Data are presented as mean $\pm S D(n=20)$. ${ }^{\star \star \star \star} P<0.0001$. The unit of measurement of MMP collapse is based on fluorescent intensity.

(Belyaeva et al., 2012; Ercal et al., 2001; Liu et al., 2017; Ranjbar et al., 2014). Therefore, damage to mitochondria with consequences such as an increase in the generation of ROS, induces oxidative stress and cell death.

\subsection{MMP assay}

As shown in Figure 3, there was a significant MMP collapse in the blood lymphocytes isolated from lead and zinc mine workers compared to control group (Figure 3a). An increase in fluorescence intensity ( $\mathrm{Rh} \mathrm{123)}$ indicates a collapse in the MMP. In the exposed and un-exposed groups, there is a no significant difference in the MMP collapse based on the comparison of age, employment and duration of work (Figures 3b-d).

MMP collapse was evaluated as one of the important parameters of mitochondrial damage. A significant collapse in MMP was observed in the blood lymphocytes isolated from the lead and zinc mine workers compared to control healthy group in our research. Changes in MMP are one of the important indicators for the evaluation of mitochondrial function. Also, the collapse of the MMP is associated with cell death induction. Therefore, mitochondria in in vitro toxicity research are usually used as an important target (Kamogashira et al., 2017; Sakamuru et al., 2016). These results are in agreement with previous studies (Chen et al., 2003; Zarei et al., 2017, 2018a).

\subsection{GSH and GSSG content assay}

The results showed that GSH content in workers in lead and zinc mine was significantly lower than un-exposed group (Figure 4a). Also, GSSG content in workers in lead and zinc mine was significantly higher than control group 
A

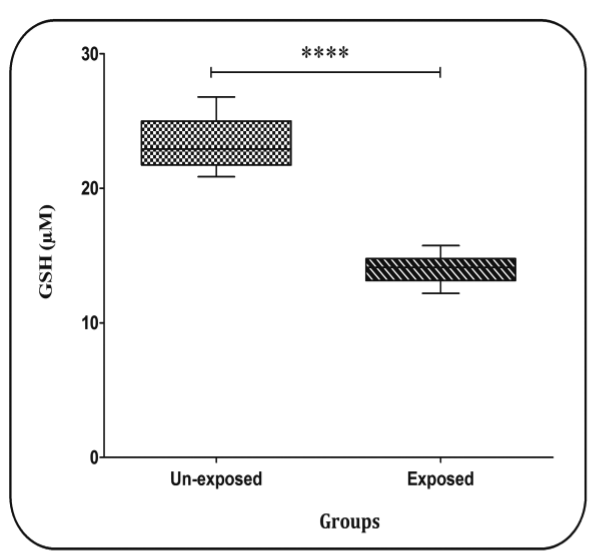

C

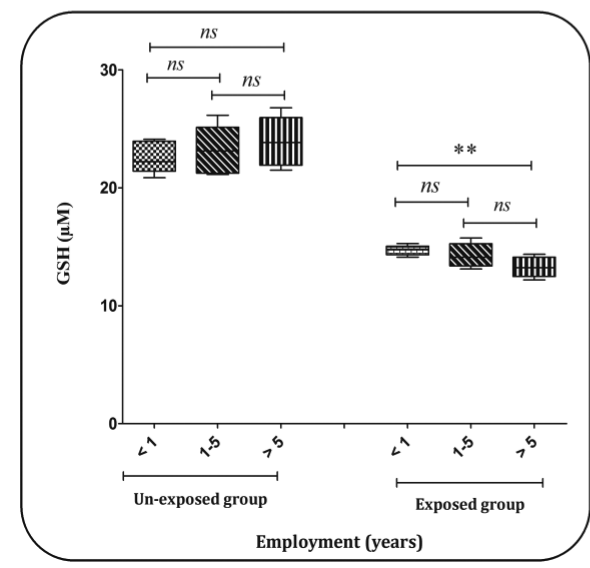

B

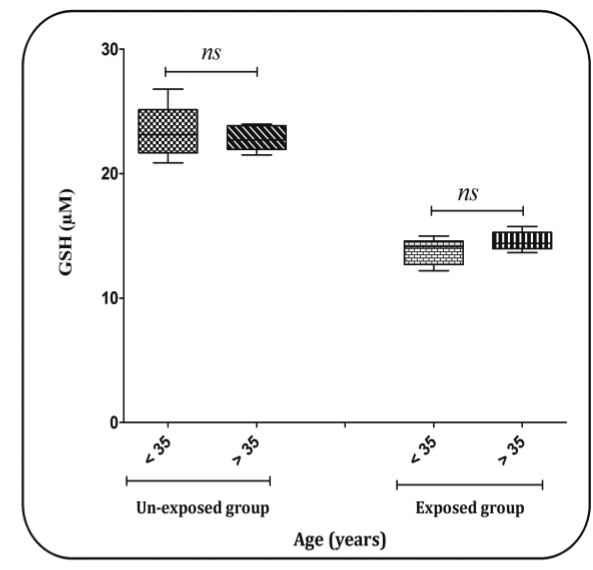

D

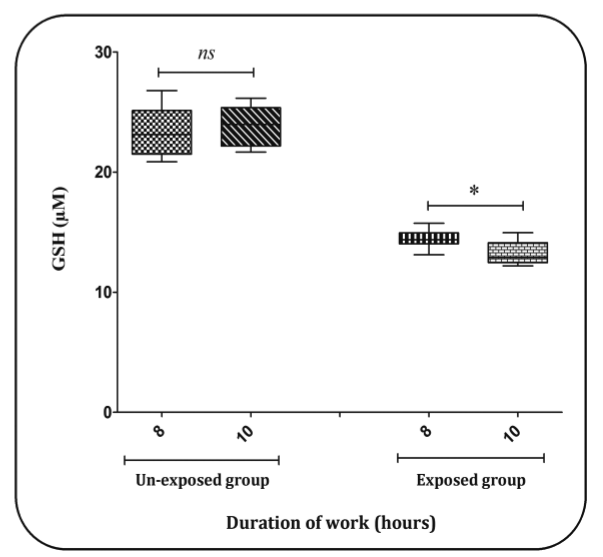

Figure 4: GSH content assay. (a) Comparison between exposure and non-exposure groups. (b) Comparison between the exposed and unexposed groups based on age (year). (c) Comparison between the exposed and unexposed groups based on employment (year). (d) Comparison between the exposed and unexposed groups based on duration of work (hours). Data are presented as mean \pm SD ( $n=20$ ). ${ }^{*} \mathrm{P}<0.05,{ }^{* *} \mathrm{P}<0.01$ and $* * * * \mathrm{P}<0.0001$.

(Figure 5a). In the exposed group, the results showed a significant difference between GSH (Figures 4c,d), and GSSG (Figures $5 \mathrm{c}, \mathrm{d}$ ) levels based on employment and duration of work.

Heavy metals such as lead and zinc have the ability to reduce GSH level which plays an important role in the antioxidant system (Conterato et al., 2013; Kasperczyk et al., 2014a, 2017; Shraideh et al., 2018; Zarei et al., 2017, 2018a). Our results indicated a decline in GSH content and increased GSSG content in blood lymphocyte taken from worker groups compared to those of control group. It has been suggested that one of the mechanisms by which the level of GSH content is reduced is due to the high tendency of lead to complex sulfhydryl groups. This group plays an important role in antioxidant defense (Kasperczyk et al., 2016; Shraideh et al., 2018).

\subsection{Lysosomal damage}

As shown in Figure 5, there was a significant lysosomal damage in the blood lymphocytes isolated from the lead and zinc mine workers compared to un-exposed group (Figure 6a). The results show that lysosomes are similar to mitochondria targeted at lead and zinc. In the exposed and un-exposed groups, there is a no significant difference in the lysosomal damage based on the comparison of age, employment and duration of work (Figures 6b-d). Disruption of the lysosomal membrane is due to hydrogen peroxide $\left(\mathrm{H}_{202}\right)$ transmission from mitochondria to cytosol and activation of the fenton reaction (Zarei et al., 2018a). Our results indicated a damage in the lysosomal membrane in miners group compared to the control group. 
A

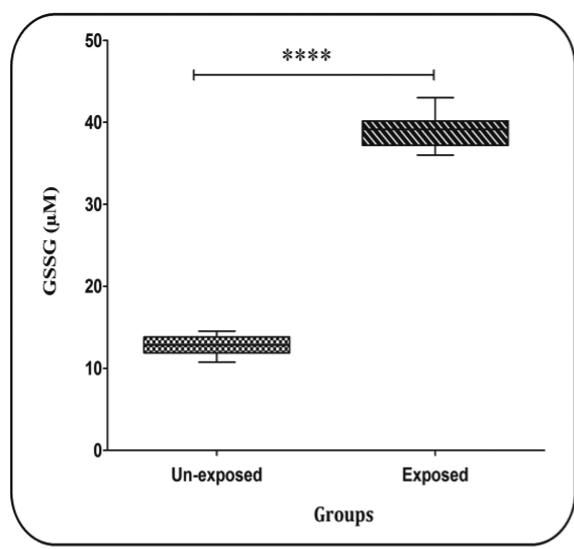

C

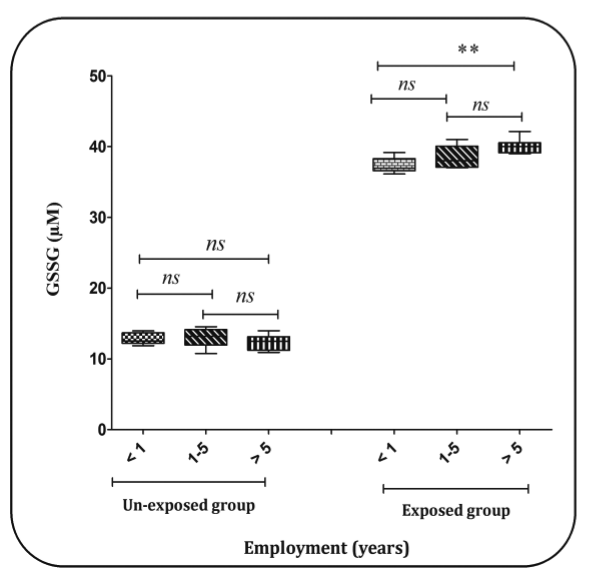

B

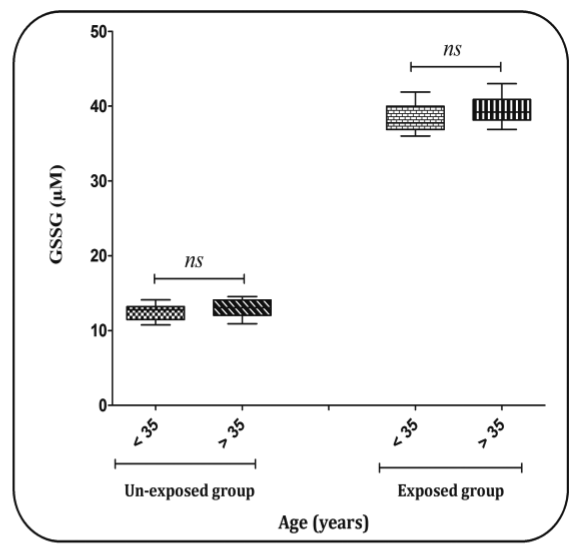

D

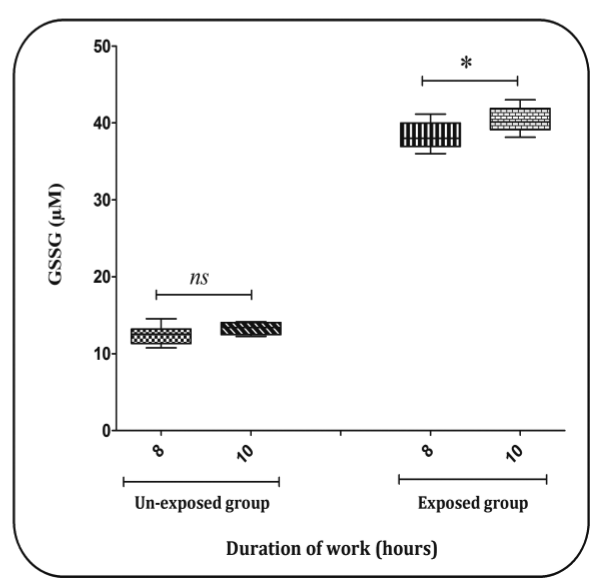

Figure 5: GSSG content assay. (a) Comparison between exposure and non-exposure groups. (b) Comparison between the exposed and unexposed groups based on age (year). (c) Comparison between the exposed and unexposed groups based on employment (year).

(d) Comparison between the exposed and unexposed groups based on duration of work (hours). Data are presented as mean \pm SD ( $n=20$ ).

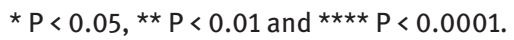

\section{Conclusion}

In conclusion, we showed that mitochondria and lysosomes are targeted at lymphocytes isolated from workers in lead and zinc mine. Also, the level of ROS, oxidative stress, collapse of MMP and GSSG content has increased in the lymphocytes isolated from lead and zinc miners.

\section{Experimental}

\section{Chemical}

2,7-dicloroflurescein diacetate (DCHF-DA), rhodamine 123 (Rh 123), MTT (3-[4,5-dimethylthiazol-2-yl]-2, 5 diphenyltetrazolium bromide) reagent and acridine orange were purchased from Sigma Chemical Co. (St. Louis, MO, USA). All other chemicals were of the highest commercial grade available.

\section{Study population}

The exposed group consisted of 32 lead-zinc mine workers ( 32 male and 0 female). 9 were smokers and 23 non-smokers; 0 were drinkers and 32 were non-drinkers; 3 donors had chronic disease or medicine consumption. The control or non-exposed group consisted of 28 volunteers, office workers, who had never been occupationally exposed to known toxic substances (28 male and 0 female), 6 among them were smokers; 0 were drinkers and 28 were non-drinkers; 2 donors had chronic disease or medicine consumption. In this study, 
A

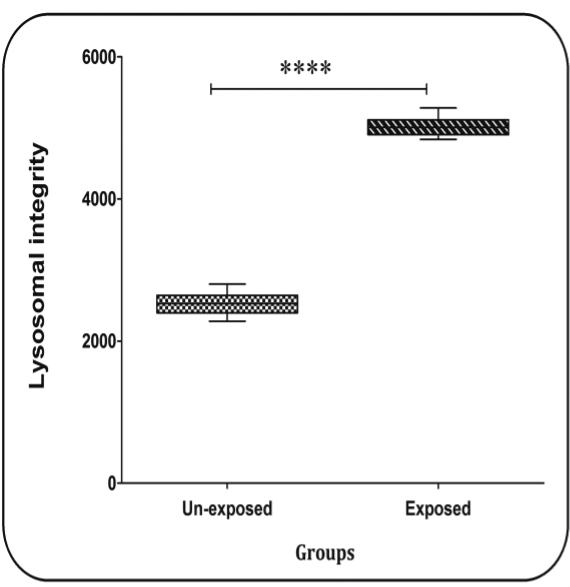

C

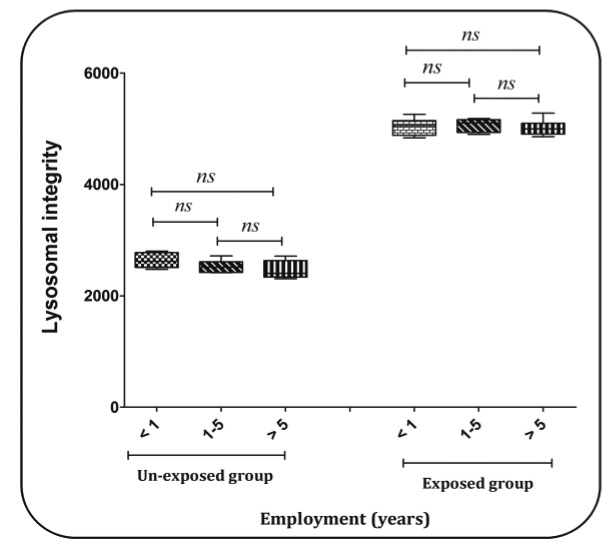

B

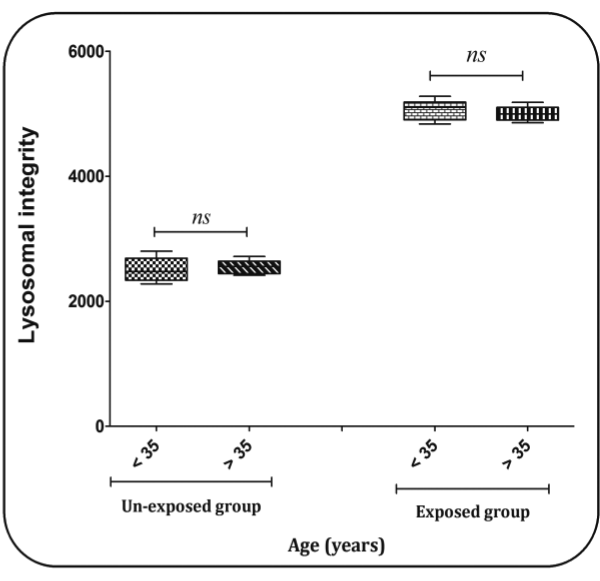

D

Figure 6: Lysosomal damage assay. (a) Comparison between exposure and non-exposure groups. (b) Comparison between the exposed and unexposed groups based on age (year). (c) Comparison between the exposed and unexposed groups based on employment (year). (d) Comparison between the exposed and unexposed groups based on duration of work (hours). Data are presented as mean \pm SD ( $n=20$ ). $\star \star \star \star P<0.0001$. The unit of measurement of lysosomal damage is based on fluorescent intensity.

exclusion criteria include smoking habits and acute and chronic disease (such as diabetes)/medicine consumption. Demography and other characteristic of donors from both groups are shown in Table 1. Finally, according to the exclusion criteria, 20 donors in each group were selected to study. The present study was approved by the ethics committee of Shahid Beheshti University of Medical Sciences as ID IR.SBMU.PHARMACY.REC.1397.202. After became aware of our study donors are asked to fill out the approval form.

\section{The blood lymphocytes isolation}

Blood samples were taken from 10 lead-zinc mine workers and 10 control workers and samples were kept in heparin syringes. Briefly, ficoll plaque $(3 \mathrm{~mL})$ was used for the isolation of lymphocytes and then centrifugation was performed (2500 rpm for $20 \mathrm{~min}$ ). The plasma layer is slowly detached and the white layer that is suspended in the lysis buffer $\left(150 \mathrm{mM} \mathrm{NH}_{4} \mathrm{Cl}, 10 \mathrm{mM} \mathrm{NaHCO}_{3}, 1 \mathrm{mM}\right.$ EDTA, $\mathrm{pH}=7.4$ ) of the erythrocyte is human lymphocytes, and then incubated for $5 \mathrm{~min}$ at $37^{\circ} \mathrm{C}$. In the next step, $10 \mathrm{~mL}$ PBS was added to the tube. Then, cells were centrifuged at $1500 \mathrm{rpm}$ and the supernatant was eliminated. Finally, cells with RPMI-1640 contain 10\% fetal bovine serum (FBS) at $2000 \mathrm{~g}$ for $7 \mathrm{~min}$ were washed twice and $1 \times 10^{6}$ cell diluted in $1 \mathrm{~mL}$ of RPMI-1640 contain $10 \%$ FBS and 1\% antibiotic (penestrep) for use in the different test (Assadian et al., 2019).

\section{Cell viability assay}

Cell viability was assayed using MTT assay. Blood lymphocytes $\left(1 \times 10^{4}\right.$ cells/well $)$ were placed in 96-well plates with RPMI 1640, supplemented with 10\% FBS and antibiotics $(50 \mathrm{U} / \mathrm{mL}$ of penicillin and $50 \mu \mathrm{g} / \mathrm{mL}$ 
Table 1: Demography and other characteristic of donors from both groups.

\begin{tabular}{lccc}
\hline & & $\begin{array}{c}\text { Exposed } \\
\text { group }\end{array}$ & $\begin{array}{c}\text { Control } \\
\text { group }\end{array}$ \\
\hline Number (n) & 32 & 28 \\
Smoking Habits & 9 & 6 \\
Medicine consumption/ & 3 & 2 \\
diseases (acute and chronic) & & 0 & 0 \\
Alcohol consumption & & 9 & 12 \\
Age & $<35$ & 11 & 8 \\
Gender & $>35$ & 32 & 28 \\
Male & 0 & 0 \\
Employment & $<1$ & 6 & 5 \\
& $1-5$ & 5 & 7 \\
Duration of work & $>5$ & 9 & 8 \\
& $8 \mathrm{~h}$ & 13 & 15 \\
& $10 \mathrm{~h}$ & 7 & 5 \\
& $12 \mathrm{~h}$ & 0 & 0 \\
& $16 \mathrm{~h}$ & 0 & 0 \\
\hline
\end{tabular}

streptomycin). In the next step, MTT ( $5 \mathrm{mg} / \mathrm{mL}$ in RPMI 1640) was added to each well and incubated for $4 \mathrm{~h}$ after which the plate was centrifuged at $1800 \mathrm{x} \mathrm{g}$ for $5 \mathrm{~min}$ at $4^{\circ} \mathrm{C}$. After careful removal of the medium, DMSO $(100 \mu \mathrm{L})$ was added to each well, and plates were shaken. Then, absorbance was recorded at $570 \mathrm{~nm}$ using microplate reader (Tecan, Rainbow Thermo, Austria) (Zhang et al., 2008).

\section{Reactive oxygen species (ROS) assay}

After isolation, human lymphocytes were incubated with $500 \mu \mathrm{L}$ of dichloro-dihydro-fluorescein Diacetate (DCFH$\mathrm{DA}$; at the concentration of $10 \mu \mathrm{M}$ ) solution for $20 \mathrm{~min}$ in $37^{\circ} \mathrm{C}$. The human lymphocytes were washed with PBS. In the final step, fluorescence intensity was measured using a fluorescence spectrophotometer (Shimadzu RF5000U, Japan) at 495 and $530 \mathrm{~nm}$ excitation and emission wavelength. The results were expressed as fluorescent intensity (DCF) per $10^{6}$ cells (Assadian et al., 2018).

\section{The mitochondrial membrane potential (MMP) assay}

At first and after removing the supernatant by centrifuging at $1000 \times \mathrm{g}$ for $5 \mathrm{~min}, 500 \mu \mathrm{L}$ of 5 rhodamine
123 (Rh123; at the concentration of $5 \mu \mathrm{M}$ ) was added to the human lymphocytes suspension and incubated for $10 \mathrm{~min}$. In the final step, fluorescence intensity was measured using a fluorescence spectrophotometer (Shimadzu RF5000U, Japan) at 470 and $540 \mathrm{~nm}$ excitation and emission wavelength. The results were expressed as fluorescent intensity (Rh 123) per $10^{6}$ cells (Zhao et al., 2010).

\section{Glutathione (GSH) and oxidized glutathione (GSSG) assay}

After isolation, TCA $10 \%(0.5 \mathrm{~mL})$ was added to the human lymphocytes and then centrifuged at 11000 RPM for $2 \mathrm{~min}$. In the next step, supernatant $(0.5 \mathrm{~mL})$ was diluted by the addition of EDTA buffer $(4.5 \mathrm{~mL})$. In addition, 1000 microliter of diluted supernatant was added to phosphate-EDTAbuffer $(2.8 \mathrm{~mL})$ and theortho-phthalaldehyde $(\mathrm{OPA} ; 100 \mu \mathrm{L})$ solution. Finally, after incubation at room temperature for $15 \mathrm{~min}$, fluorescence intensity was assayed at the $\lambda \mathrm{Ex}=350 \mathrm{~nm}$ and $\lambda \mathrm{Em}=420$ using a fluorescence spectrophotometer (Shimadzu RF5000U, Japan) (Assadian et al., 2019; Hilf and Hissin, 1976).

\section{Lysosomal damage assay}

To perform this test, $1000 \mu \mathrm{L}$ of acridine orange at the concentration of $5 \mu \mathrm{M}$ was added to the human lymphocytes $(100 \mu \mathrm{L})$ and incubated for $10 \mathrm{~min}$ in $37^{\circ} \mathrm{C}$. After washing the lymphocytes, the fluorescence intensity (acridine orange) was assayed at the $\lambda \mathrm{Ex}=470 \mathrm{~nm}$ and $\lambda \mathrm{Em}=540$ using a fluorescence spectrophotometer (Shimadzu RF5000U, Japan).

\section{Statistical analysis results}

Data were analyzed using SPSS software version 20 and GraphPad Prism 5 (Graphpad Software, La Jolla, CA). The Kolmogorov-Smirnov test was used to examine the distribution of data. Due to the normal distribution of data, one-way ANOVA test, followed by the post hoc Tukey test and independent sample T-Test were used to analyze the data. Values less than 0.05 were considered significant levels.

Conflict of interest: The authors declare that there is no conflict of interest. 
Acknowledgments: The data provided in this article was extracted from the Pharm D. thesis of Dr. Mahshid Soltani. The thesis was conducted under supervision of Prof. Jalal Pourahmad at Department of Toxicology and Pharmacology, Faculty of Pharmacy, Shahid Beheshti University of Medical Sciences, Tehran, Iran.

\section{References}

Akram Z., Riaz S., Kayani M.A., Jahan S., Ahmad M.W., Ullah M.A., et al., Lead induces DNA damage and alteration of ALAD and antioxidant genes mRNA expression in construction site workers. Arch. Environ. Occup. H., 2018, 1-8.

Assadian E., Dezhampanah H., Seydi E., Pourahmad J., Toxicity of $\mathrm{Fe}_{2} \mathrm{O}_{3}$ nanoparticles on human blood lymphocytes. J. Biochem. Mol. Toxicol., 2019, 33(6), e22303.

Assadian E., Zarei M.H., Gilani A.G., Farshin M., Degampanah H., Pourahmad J., Toxicity of copper oxide (CuO) nanoparticles on human blood lymphocytes. Biol. Trace Elem. Res., 2018, 184, 350-357.

Basit S., Karim N., Munshi A.B., Occupational lead toxicity in battery workers. Pak. J. Med. Sci., 2015, 31(4), 775-780.

Belyaeva E.A., Sokolova T.V., Emelyanova L.V., Zakharova I.O., Mitochondrial electron transport chain in heavy metal-induced neurotoxicity: effects of cadmium, mercury, and copper. Sci. World J., 2012, 2012,136063.

Chen L., Yang X., Jiao H., Zhao B., Tea catechins protect against lead-induced ROS formation, mitochondrial dysfunction, and calcium dysregulation in PC12 cells. Chem. Res. Toxicol., 2003, 16(9), 1155-1161.

Conterato G.M., Bulcao R.P., Sobieski R., Moro A.M., Charao M.F., de Freitas F.A., et al., Blood thioredoxin reductase activity, oxidative stress and hematological parameters in painters and battery workers: relationship with lead and cadmium levels in blood. J. Appl. Toxicol., 2013, 33(2), 142-50.

Dan Dunn J., Alvarez L.A., Zhang X., Soldati T., Reactive oxygen species and mitochondria: A nexus of cellular homeostasis. Redox Biol., 2015, 6, 472-845.

Ercal N., Gurer-Orhan H., Aykin-Burns N., Toxic metals and oxidative stress part I: mechanisms involved in metal-induced oxidative damage. Curr. Top. Med. Chem., 2001, 1(6), 529-539.

Fedotcheva T., Shimanovskii N., Kruglov A., Teplova V., Fedotcheva N., Role of mitochondrial thiols of different localization in the generation of reactive oxygen species. Biochem (Mosc). Suppl. Ser. A Membr. Cell Biol., 2012, 6(1), 92-99.

Fracasso M.E., Perbellini L., Solda S., Talamini G., Franceschetti P., Lead induced DNA strand breaks in lymphocytes of exposed workers: role of reactive oxygen species and protein kinase $C$. Mutat. Res., 2002, 515(1-2), 159-169.

Ghanwat G., Patil A.J., Patil J., Kshirsagar M., Sontakke A., Ayachit R.K., Effect of Vitamin C Supplementation on Blood Lead Level, Oxidative Stress and Antioxidant Status of Battery Manufacturing Workers of Western Maharashtra, India. J. Clin. Diagn. Res., 2016, 10(4), Bc08-11.

Hilf R., Hissin P., A fluorometric method for determination of oxidized and reduced glutathione in tissues. Anal. Biochem., 1976, 74(1), 214-226.
Hsieh N.H., Chung S.H., Chen S.C., Chen W.Y., Cheng Y.H., Lin Y.J., et al., BMC Public Health. BMC Public Health, 2017, 17(1), 89.

Kalahasthi R., Barman T., Assessment of Lead Exposure and Urinary-delta-aminolevulinic Acid Levels in Male Lead Acid Battery Workers in Tamil Nadu, India. J. Health Pollut., 2018, 8(17), 6-13.

Kamogashira T., Hayashi K., Fujimoto C., Iwasaki S., Yamasoba T., Functionally and morphologically damaged mitochondria observed in auditory cells under senescence-inducing stress. NPJ Aging Mech. Dis., 2017, 3, 2.

Kasperczyk S., Dobrakowski M., Kasperczyk A., Machnik G., Birkner E., Effect of $\mathrm{N}$-acetylcysteine administration on the expression and activities of antioxidant enzymes and the malondialdehyde level in the blood of lead-exposed workers. Environ. Toxicol. Pharmacol., 2014a, 37(2), 638-647.

Kasperczyk S., Dobrakowski M., Kasperczyk A., Nogaj E., Boron M., Szlacheta Z., et al., alpha-Tocopherol supplementation and the oxidative stress, homocysteine, and antioxidants in lead exposure. Arch. Environ. Occup. Health., 2017, 72(3), 153-158.

Kasperczyk S., Dobrakowski M., Kasperczyk A., Romuk E., Rykaczewska-Czerwińska M., Pawlas N., et al., Effect of N-acetylcysteine administration on homocysteine level, oxidative damage to proteins, and levels of iron ( $\mathrm{Fe}$ ) and Fe-related proteins in lead-exposed workers. Toxicol. Ind. Health, 2016, 32(9), 1607-1618.

Kasperczyk S., Dobrakowski M., Kasperczyk J., Ostalowska A., Zalejska-Fiolka J., Birkner E., Beta-carotene reduces oxidative stress, improves glutathione metabolism and modifies antioxidant defense systems in lead-exposed workers. Toxicol. Appl. Pharmacol., 2014b, 280(1), 36-41.

Li X., Fang P., Mai J., Choi E.T., Wang H., Yang X.F., Targeting mitochondrial reactive oxygen species as novel therapy for inflammatory diseases and cancers. J. Hematol. Oncol., 2013, 6,19.

Liu B., Qin H., Zhang B., Shi T., Cui Y., Enhanced oxidative stress by lead toxicity retards cell survival in primary thyroid cells. Int. J. Clin. Exp. Med., 2017, 10(3), 4590-4597.

Plum L.M., Rink L., Haase H., The essential toxin: impact of zinc on human health. Int. J. Environ. Res. Public. Health, 2010, 7(4), 1342-1365.

Qu C.S., Ma Z.W., Yang J., Liu Y., Bi J., Huang L., Human exposure pathways of heavy metals in a lead-zinc mining area, Jiangsu Province, China. PLoS One, 2012, 7(11), e46793.

Qu W., Du G.L., Feng B., Shao H., Effects of oxidative stress on blood pressure and electrocardiogram findings in workers with occupational exposure to lead. J. Int. Med. Res., 2019, 47(6), 2461-2470.

Ranjbar A., Ghasemi H., Rostampour F., The role of oxidative stress in metals toxicity; mitochondrial dysfunction as a key player. Galen Med. J., 2014, 3(1), 2-13.

Ricco M., Cattani S., Signorelli C., Zinc exposure for female workers in a galvanizing plant in Northern Italy. Int. J. Occup. Med. Env., 2018, 31(1), 113-124.

Sakamuru S., Attene-Ramos M.S., Xia M., Mitochondrial Membrane Potential Assay. Methods Mol. Biol., 2016, 1473,17-22.

Shraideh Z., Badran D., Hunaiti A., Battah A., Association between occupational lead exposure and plasma levels of selected oxidative stress related parameters in Jordanian automobile workers. Int. J. Occup. Med. Env., 2018, 31(4), 517-525. 
Steffensen I.L., Mesna O.J., Andruchow E., Namork E., Hylland K., Andersen R.A., Cytotoxicity and accumulation of $\mathrm{Hg}, \mathrm{Ag}, \mathrm{Cd}$, $\mathrm{Cu}, \mathrm{Pb}$ and $\mathrm{Zn}$ in human peripheral $\mathrm{T}$ and $\mathrm{B}$ lymphocytes and monocytes in vitro. Gen. Pharmacol., 1994, 25(8), 1621-1633.

Zarei M.H., Hosseini Shirazi S.F., Aghvami M., Salimi A., Pourahmad J., Analysis of cytotoxic effects of nickel on human blood lymphocytes. Toxicol. Mech. Method., 2018a, 28(2), 79-86.

Zarei M.H., Pourahmad J., Aghvami M., Soodi M., Nassireslami E., Lead acetate toxicity on human lymphocytes at non-cytotoxic concentrations detected in human blood. Main Group Met. Chem., 2017, 40(5-6), 105-112.
Zhang Q., Zhao X.-H., Wang Z.-J., Flavones and flavonols exert cytotoxic effects on a human oesophageal adenocarcinoma cell line (OE33) by causing G2/M arrest and inducing apoptosis. Food Chem. Toxicol., 2008, 46(6), 2042-2053.

Zhao Y., Ye L., Liu H., Xia Q., Zhang Y., Yang X., et al., Vanadium compounds induced mitochondria permeability transition pore (PTP) opening related to oxidative stress. J. Inorg. Biochem., 2010, 104(4), 371-378.

Zorov D.B., Juhaszova M., Sollott S.J., Mitochondrial reactive oxygen species (ROS) and ROS-induced ROS release. Physiol. Rev., 2014, 94(3), 909-950. 